\title{
Collaborative Spectrum Sensing for Cognitive Radio: Diversity Combining Approach
}

\author{
Oscar Filio-Rodriguez ${ }^{1}$, V. Kontorovich ${ }^{2}$, Serguei Primak ${ }^{1}$, F. Ramos-Alarcon ${ }^{2}$ \\ ${ }^{1}$ Department of Electrical and Computing Engineering, University of Western Ontario, London, Canada \\ ${ }^{2}$ Electrical Engineering Department, Research and Advanced Studies Center, Mexico City, Mexico. \\ E-mail:valeri@cinvestav.mx \\ Received August 5, 2010; revised September 2, 2010; accepted January 15, 2011
}

\begin{abstract}
In this paper it is shown that cyclostationary spectrum sensing for Cognitive Radio networks, applying multiple cyclic frequencies for single user detection can be interpreted (with some assumptions) in terms of optimal incoherent diversity addition for "virtual diversity branches" or SIMO radar. This approach allows proposing, by analogy to diversity combining, suboptimal algorithms which can provide near optimal characteristics for the Neyman-Pearson Test (NPT) for single user detection. The analysis is based on the Generalized Gaussian (Klovsky-Middleton) Channel Model, which allows obtaining the NPT noise immunity characteristics: probability of misdetection error $\left(P_{M}\right)$ and probability of false alarm $\left(P_{f a}\right)$ or Receiver Operational Characteristics (ROC) in the most general way. Some quasi-optimum algorithms such as energetic receiver and selection addition algorithm are analyzed and their comparison with the noise immunity properties (ROC) of the optimum approach is provided as well. Finally, the diversity combining approach is applied for the collaborative spectrum sensing and censoring. It is shown how the diversity addition principles are applied for distributed detection algorithms, called hereafter as SIMO radar or distributed SIMO radar, implementing Majority Addition (MA) approach and Weighted Majority Addition (WMA) principle.
\end{abstract}

Keywords: Spectrum Sensing, Cognitive Radio, Diversity Combining, Collaborative Sensing, Majority Diversity Addition, Sequential Analysis

\section{Introduction}

Spectrum sensing is one of the most important elements for the functioning of Cognitive Radio (CR) networks.

As it is well known [1], CR networks are made up of primary users (PU) which have "legal" use of certain frequency bands and secondary or cognitive users (CU), located in different space-distributed cells, which share the same frequencies as the PU in a part-time fashion.

The CU produce undesired interferences to the PU and so they are allowed to share the same spectrum with the PU if and only if the Quality of Service (QoS) degradation provoked to the PU does not reach a pre-established level.

The cognitive users have to make first a spectrum sensing in order to determine whether the primary users are "on" or "off" and then "adapt" their transmission rate and transmission power in order to avoid producing

\footnotetext{
${ }^{1}$ Actually it is not a necessary condition for CU to have access to the frequencies allocated to PU [2].
}

harmful interferences to the PU or take advantage of the "spectrum holes" free of PU [1,2], etc.

The common approaches are based on the Interference Temperature (IT) and power spectrum estimations, energy detection and cyclostationary feature detection (see [1-4] and the references therein). The last one was first proposed in [5] and generalized for multiple cyclic frequencies at [4].

It is worth mentioning here that the cyclostationarity, as phenomenon, is not a recent development at all (see for example [6]) but effective tests for indication of second order cyclostationarity using a Neyman-Pearson type test was proposed not long ago. Its natural generalization for multiple cyclic frequencies was recently proposed for PU identification in CR networks [4].

As it was already mentioned in $[4,5]$ the cyclostationarity is present, practically, in many communication signals: multiple cyclic frequencies may be related to symbol rate, guard periods (as in the case of OFDM systems), etc. 
In the following it will be shown that single-user detection algorithms (in the form of expected value estimation of the cyclic autocorrelation) can be interpreted as an specific form of the mixed frequency-delay incoherent "diversity combining" block with the number of virtual "branches" equal to the product numbers of cyclic frequencies and time delays; it can be also called as a SIMO radar. This is the main difference between this paper and the material presented in $[4,5]$.

Based on practical reasons, it is possible to assume that these "branches" suffer from fading which in the general case can be modeled with the help of generalized Gaussian statistics, or Klovsky-Middleton model, (see for example [7]).

Moreover, in the following, depending on the frequency and delay diversity parameters, fading in these branches or antennas is assumed as non-homogeneous, homogeneous and totally correlated (the latter was considered at [4] for flat Rayleigh fading) or statistically independent. Certainly those last two marginal cases are assumed in the following only in order to obtain tractable analytical results; generalized analysis for correlated branches, based on the statistical description of Gaussian quadratic forms (see $[8,9]$, etc.) will be considered elsewhere later on, but one important special case of correlated branches is considered in the following as well.

It is worth mentioning that the concept of "diversity approach" for multiple cyclic detection is useful not only for effective development of quasi-optimal approaches, but also allows to consider the necessary "trade-off" between the number of delays and cyclic frequencies for the detection procedure and the statistical dependency of the corresponding "diversity branches" in order to fulfill the noise immunity or "Receiver Operating Characteristics" (ROC) requirements.

Moreover, in the following, it will be shown that the "diversity" concept for spectrum sensing is rather constructive for the analysis of collaborative sensing as well (see [9], etc.). In [9] the collaboration is tackled in a rather different way, than in the following. For the latter the set of secondary users (SU) being collaborating between themselves or operating through a Fusion Center (FC) can be interpreted as virtual branches (antennas) of the distributed detection system, which can apply NPT detection technique or Sequential Analysis methods.

This distributed system is nothing else as a distributed SIMO radar, where virtual receiving branches are affected by statistically independent flat fading (the above mentioned incoherent combining algorithm at SU is also working as an optimum SIMO radar, but not in a distributed fashion). See also some examples at [10,11], etc.

\footnotetext{
${ }^{2}$ Those issues were tackled comprehensively in the $60^{\text {th }}-70^{\text {th }}$ of the last century by many authors. Here we would like to distinguish D. Klovsky [8], D. Middleton [16], P. Beckman [17], etc. Details can be found at [7].
}

for calculation of its noise immunity properties.

Regularly proposed counting rules [2,12-14] for operating at FC can be also interpreted as a special case of quasi-optimum incoherent diversity addition (see MA algorithm in the following) and can be modified in order to approach its ROC properties to the optimum SIMO radar case (see the WMA algorithm in the following).

In this paper only the novel theoretical material, which is the kernel of a deeper and original insight into the Spectrum Sensing problem, is presented. Some simulations related to this problem have been included in [15]; however, comprehensive and thorough simulations are reserved for another work of the authors (a book chapter already in process for publication).

The paper is organized as follows. Section 2 briefly presents some fundamental results concerned to the Generalized Gaussian (GG) channel modeling. Section 3 is totally dedicated to single user multiple cyclic frequency detection and its relation to incoherent optimum diversity combining. In section 4 the noise immunity of the NPT for the GG channel is analyzed. In Section 5 some suboptimal algorithms for multiple frequency cyclostationary sensing are considered. Here some discussion of the results is presented as well. Section 6 is totally dedicated to collaborative sensing issues. Conclusions will be presented at Section 7.

\section{Generalized Gaussian (Klovsky-Middleton) Channel Model}

Basically most of the existing fading channel models are based on the concept of the module and phase of the random vector with Gaussian Probability Density Functions (PDF) for orthogonal statistically independent quadrature components " $x$ " and " $y$ ", i.e., $[7,8]$ :

$$
W(x, y)=\frac{1}{2 \pi \sigma_{x} \sigma_{y}} \exp \left[-\frac{\left(x-m_{x}\right)^{2}}{2 \sigma_{x}^{2}}-\frac{\left(y-m_{y}\right)^{2}}{2 \sigma_{y}^{2}}\right]
$$

where $\sigma_{x}^{2}, \sigma_{y}^{2}$ and $m_{x}, m_{y}$ are variances and expectations of the " $x$ " and " $y$ " quadrature components respectively.

Then, defining the module $\gamma=\sqrt{x^{2}+y^{2}}$ and the phase of the random vector $\varphi=\arctan \frac{y}{x}$, one can get:

$$
W(\gamma)=\frac{\gamma}{2 \pi \sigma_{x} \sigma_{y}} \int_{0}^{2 \pi} \exp \left[-\frac{\left(\gamma \cos \varphi-m_{x}\right)^{2}}{2 \sigma_{x}^{2}}-\frac{\left(\gamma \sin \varphi-m_{y}\right)^{2}}{2 \sigma_{y}^{2}}\right] d \varphi
$$

From (2) it is possible to obtain various representations for $W(\gamma)$, which actually depend on four parameters: $\left\{m_{x}, m_{y}\right\}$ and $\left\{\sigma_{x}^{2}, \sigma_{y}^{2}\right\},[7,8]$. For this reason in the following the term "four parametric distribution" is used, and the rest of this section corresponds to [8]. 
Hereafter the following two equivalent forms for the four-parametric distribution $W(\gamma)^{3}$ will be used:

$$
\begin{gathered}
W(\gamma)=\frac{\gamma}{\sigma_{x} \sigma_{y}} \exp \left[-\frac{m_{y}^{2}+\gamma^{2}}{2 \sigma_{y}^{2}}-\frac{m_{x}^{2}}{2 \sigma_{x}^{2}}\right] \\
\cdot \sum_{k=0}^{\infty} \frac{H_{2 k}(\alpha)}{(2 k) ! ! 2^{k}} \gamma^{k}\left(\frac{\sigma_{y}^{2}}{m_{y}}\right)^{k}\left(\frac{1}{\sigma_{x}^{2}}-\frac{1}{\sigma_{y}^{2}}\right)^{k} I_{k}\left(\frac{m_{y}}{\sigma_{y}^{2}}\right) \\
W(\gamma)=\sum_{k=0}^{\infty} \frac{R^{k}}{k !} \sigma^{2 k} \frac{\partial^{2 k}}{\partial m_{I}^{k} \partial m_{I I}^{k}}\left\{\frac{\gamma}{\sigma^{2}}\right. \\
\left.\exp \left[-\frac{\gamma^{2}+m_{I}^{2}+m_{I I}^{2}}{2 \sigma^{2}}\right] I_{0}\left(\frac{\gamma}{\sigma^{2}} \sqrt{m_{I}^{2}+m_{I I}^{2}}\right)\right\}
\end{gathered}
$$

where $I_{0}(\mathrm{z})$ is the modified Bessel function of order zero

$$
\begin{aligned}
& \text { [18], } m_{I}=\frac{m_{x}+m_{y}}{\sqrt{2}}, \quad m_{I I}=\frac{m_{x}-m_{y}}{\sqrt{2}}, \quad \sigma^{2}=\frac{\sigma_{x}^{2}+\sigma_{y}^{2}}{2}, \\
& R=\frac{\sigma_{y}^{2}-\sigma_{x}^{2}}{\sigma_{x}^{2}+\sigma_{y}^{2}}, \quad \alpha=\frac{m_{x}}{\sqrt{2\left(\frac{1}{\sigma_{x}^{2}}-\frac{1}{\sigma_{y}^{2}}\right)} \sigma_{y}^{2}}\left(\sigma_{x}^{2} \leq \sigma_{y}^{2}\right) .
\end{aligned}
$$

Other forms (see for example [7]) follow from the way the integrand in (2) is calculated, but they are not applied in the following.

Beckman, Hoyt, Rice, Rayleigh and truncated Gaussian distributions follow from (3) directly.

Let us introduce the new parameters:

$$
\begin{gathered}
q^{2}=\frac{m_{x}^{2}+m_{y}^{2}}{\sigma_{x}^{2}+\sigma_{y}^{2}}, \quad \beta^{2}=\frac{\sigma_{x}^{2}}{\sigma_{y}^{2}}, \quad \gamma_{0}^{2}=m_{x}^{2}+m_{y}^{2}, \\
\left\langle\gamma^{2}\right\rangle=\gamma_{0}^{2}+\sigma_{x}^{2}+\sigma_{y}^{2}, \quad \varphi_{0}=\arctan \frac{m_{y}}{m_{x}}
\end{gathered}
$$

Beckman distribution follows from (3) when $m_{y}=0, \gamma_{0}$ $=\left|m_{x}\right|$, while Hoyt PDF appears, when $\sigma_{x}^{2} \neq \sigma_{y}^{2} \quad \gamma_{0}=$ $m_{x}=m_{y}=0$. Rayleigh PDF follows when $\gamma_{0}=0$ and $m_{x}=$ $m_{y}=0$; truncated Gaussian when additionally to the latter $\sigma_{x}^{2} \rightarrow 0$.

That is why (3) is named as Generalized Gaussian model. Next it is easy to find the parameter " $m$ " for equivalent Nakagami distribution [19]:

$$
m=\frac{\left(1+\beta^{2}\right)^{2}\left(1+q^{2}\right)^{2}}{2\left[1+\beta^{4}+2 q^{2}\left(1+\beta^{2}\right)\left(\beta^{2} \cos ^{2} \phi_{0}+\sin ^{2} \phi_{0}\right)\right]}
$$

It is worth mentioning here that Nakagami distribution is only an approximation for the four-parameter case, but mainly it adequately represents the "dynamics" of the variation of the four-parameter PDF functional form.

${ }^{3}$ Taking into account, that in this paper the incoherent diversity combining will be applied, the PDF of the phase $\varphi$ is not presented hereafter.

\section{Single User Multiple Cyclic Frequency Detection}

The cyclostationary (CL) properties of the communication signals have been already widely investigated and applied (see $[4,5,20]$ etc.).

For the case of PU the signal shapes are known a- priori, and so their cyclic frequencies of interest are known as well. Following here the material of [4], let us introduce the set $A=\left\{\alpha_{n}\right\}_{1}^{P}$ for cyclic frequencies of interest and let $N=\sum_{n=1}^{P} N_{n}$ be the numbers of integers for time delays $\tau$ for the autocovariance function calculus for each cyclic frequency from $A$ (here $P$ denotes the number of cyclic frequencies).

Thus, the estimation of the autocovariance function is [4]:

$$
\hat{R}_{x x^{*}}(\alpha, \tau)=\frac{1}{M} \sum_{l=1}^{M} x(l) x *(l+\tau) \exp (-j 2 \pi \alpha l \tau),
$$

where the time delay $\tau$ is an integer and is fixed, the cyclic frequency $\alpha$ is fixed as well, $M$ is the number of observations at $(5)$ and $x(l)$ is an input complex sample, with $x^{*}(l)$ being its complex conjugate.

Representing the complex exponent in (5) in a trigonometric form and assuming that $x(l)$ is a sample of the ergodic stochastic process, one can easily see that when $M \gg 1$ or the time of analysis $T$ is much more than one, the estimations $\hat{R}_{x x^{*}}$ are nothing else but estimations of the complex Fourier coefficients for fixed $\alpha$ and $\tau$ (see also [6]).

If one forms a complex vector of (5) for different $\alpha$ and $\tau$, the Generalized Maximum Likelihood Ratio (GMLR) for its estimation (assuming asymptotic Gaussianity of the observation) is well known (see for example $[4,8,21])$ :

$$
\hat{r}_{x x^{*}} \hat{\varepsilon}^{-1} \hat{r}_{x x^{*}}^{T} \geq \Lambda_{0}
$$

where $\hat{r}_{x x^{*}}$ is a complex vector of estimations of the Fourier coefficients (F-Coefficients); $\hat{\varepsilon}$ is a $2 N \times 2 N$ covariance matrix of $\hat{r}_{x x^{*}}$ (in the non-asymptotic case generally those coefficients are correlated),

$$
N=\sum_{n=1}^{P} N_{n}
$$

Let us define an estimation of each " $j$ " complex F- coefficient as

$$
\hat{V}_{j}=V_{j}+\widetilde{V}_{j},
$$

where $V_{j}, \widetilde{V}_{j}$ are real and imaginary parts of $\hat{V}_{j}$; here it is assumed that in the estimation process necessarily takes place $n(t)$ - the additive white Gaussian noise (AWGN) with intensity $N_{0}$ equal for all $j$. 
It is well known that "true" F-Coefficients are not correlated, but their estimations for finite " $M$ " and corrupted by the noise are not correlated only asymptotically, when $M$ or $T$ (or both) are much more than one. With this assumption, applied systematically in the following, (6) can be significantly simplified taking into account the total Gaussianity of the terms in (8) [8], (see also [4]):

$$
\sum_{\tau=1}^{N} \sum_{\alpha=1}^{P} \varepsilon_{\tau, \alpha}^{-1} \hat{V}^{2}(\tau, \alpha)=\sum_{i=1}^{Q}\left|\hat{V}_{i}\right|^{2} \varepsilon_{i}^{-1}, Q=N P,
$$

where $\varepsilon_{i}^{-1}=\operatorname{diag}\left\{\frac{1}{2 h_{1}^{2}}, \cdots, \frac{1}{2 h_{Q}^{2}}\right\}$.

Finally the left side in (6) can be represented in the way:

$$
\sum_{i=1}^{Q} \frac{V_{i}^{2}+\widetilde{V}_{i}^{2}}{2 h_{i}^{2}}
$$

where $h_{i}^{2}=2 E_{i} / N_{0},\left|\hat{V}_{i}\right|^{2}=V_{i}^{2}+\widetilde{V}_{i}^{2}, E_{i}=P_{i} T$ and $P_{i}$ is the average power of each F-coefficient (fading is not considered here).

One can see that the algorithms (8), (8a) are nothing else but an optimum incoherent quadratic diversity combining of $Q$ total virtual "branches", where $\frac{1}{2 h_{i}^{2}}$ are weighting coefficients for each branch, generally related to inhomogeneous conditions for combining.

Note that quadratic combining to obtain the NPT can be presented in the way:

$$
\sum_{i=1}^{Q} \frac{\left|\hat{V}_{i}\right|^{2}}{2 h_{i}^{2}} \geq \Lambda_{0},
$$

where $\Lambda_{0}$ is a detection threshold.

Formula (9) is not only a formal analogy with diversity addition or SIMO radar test: it is an essential reflection of the analogy between the autocovariance estimation and diversity combining of statistically independent data (see also [22]).

So, in absence of fading, all branches are asymptotically statistically independent.

In presence of fading $\hat{V}_{i}^{2}$ can be statistically independent as well, but also might be totally correlated in scenarios of flat fading both in frequency and time domains. Both cases will be considered while noise immunity of this single user multiple cyclic frequency algorithm will be analyzed (see next section).

\section{Noise Immunity of the Algorithm (9) in Generalized Gaussian Channels}

It is well known [23] that the Neyman-Pearson Test (NPT)

${ }^{4}$ In the following the module sign will be omitted. in terms of hypothesis testing, can be formulated as following:

$$
\begin{aligned}
& H_{0}: \sum_{i=1}^{Q} \frac{\left|\hat{V}_{i}\right|^{2}}{2 h_{i}^{2}}=n(t), \\
& H_{1}: \sum_{i=1}^{Q} \frac{\left|v_{i}\right|^{2}}{2 h_{i}^{2}}+n(t)
\end{aligned}
$$

here $v_{i}^{2}$ are "true" F-coefficients, $n(t)$ - white Gaussian noise with intensity $N_{0}$.

For simplicity, in the following let us suppose that all $1 / 2 h_{i}^{2}$ are the same and inhomogeneous features of the virtual branches will be addressed to different $\left\langle\gamma_{i}^{2}\right\rangle=$ $\gamma_{0}^{2}+\sigma_{x_{i}}^{2}+\sigma_{y_{i}}^{2}$. It means that in (10) one has to consider only the routine form for quadratic combining ${ }^{4}$ :

$$
z=\sum_{i=1}^{Q}\left|\hat{V}_{i}\right|^{2}
$$

As it is well known, the NPT is characterized by $P_{f a}$ and $P_{M}$ which are respectively the probability of false alarm and the probability of misdetection error [23].

In absence of fading, the " $z$ " is formed by squares of the normally distributed components and its PDF for different hypothesis can be defined in the way $[8,23]$ :

$$
\begin{aligned}
& H_{0}: \chi_{2 Q}^{2}(z) \quad \text { - central chi - square PDF } \\
& H_{1}: \chi_{2 Q}^{2}\left(z, \sum_{i=1}^{Q}\left\langle\hat{V}_{i}^{2}\right\rangle\right) \text { - non - central chi - square PDF }
\end{aligned}
$$

where $\sum_{i=1}^{Q}\left\langle\hat{V}_{i}^{2}\right\rangle$ is the expectation of the sum of $\hat{V}_{i}^{2}$ and is a parameter of the noncentral chi-square distribution [11].

It is worth to notice that in presence of fading, the functional forms for these distributions will differ depending on the scenarios for GG channel model and will be considered in the following.

\subsection{Statistically Independent Virtual Branches with Flat Generalized Gaussian Fading in Each Branch}

Let us assume that each $\hat{V}_{\dot{i}}^{2}$, see $(8,8 \mathrm{a})$, is: $\hat{V}_{i}^{2}=\hat{x}_{i}^{2}+\hat{y}_{i}^{2}$, where, $\hat{x}_{i}=x_{i} V_{i}, \hat{y}_{i}=y_{i} V_{i}$ and $\left\{x_{i}, y_{i}\right\}$ are quadrature Gaussian components of the GG fading model. Here we have to notice that for both hypothesis each quadrature components in $\hat{V}_{i}^{2}$ now are Gaussian as well, as before, but their means are not equal and their variances are arbitrary.

Now, if

$$
z=\sum_{i=1}^{Q} V_{i}^{2}+\widetilde{V}_{i}^{2}
$$

the routine procedure for calculus of the noise immunity can be applied $[7,8,23]$, etc. 
Particularly for hypothesis $H_{0}$ :

$$
\begin{aligned}
& M\left\{V_{i}\right\}=m_{x_{i}} \sqrt{\frac{2 E_{i}}{N_{0}}} \frac{1}{2 h_{x_{i}}^{2}} \sqrt{\frac{2 h_{x_{i}}^{2}}{1+2 h_{x_{i}}^{2}}} \\
& M\left\{\tilde{V}_{i}\right\}=m_{y_{i}} \sqrt{\frac{2 E_{i}}{N_{0}}} \frac{1}{2 h_{y_{i}}^{2}} \sqrt{\frac{2 h_{y_{i}}^{2}}{1+2 h_{y_{i}}^{2}}} \\
& D\left\{V_{i}\right\}=\frac{2 h_{x_{i}}^{2}}{1+2 h_{x_{i}}^{2}}, \quad D\left\{\tilde{V}_{i}\right\}=\frac{2 h_{y_{i}}^{2}}{1+2 h_{y_{i}}^{2}}
\end{aligned}
$$

For hypothesis $H_{1}$ :

$$
\begin{aligned}
& M\left\{V_{i}\right\}=m_{x_{i}} \sqrt{\frac{2 E_{i}}{N_{0}}} \sqrt{\frac{2 h_{x_{i}}^{2}}{1+2 h_{x_{i}}^{2}}} \\
& M\left\{\tilde{V}_{i}\right\}=m_{y_{i}} \sqrt{\frac{2 E_{i}}{N_{0}}} \sqrt{\frac{2 h_{y_{i}}^{2}}{1+2 h_{y_{i}}^{2}}} \\
& D\left\{V_{i}\right\}=2 h_{x_{i}}^{2}, \quad D\left\{\tilde{V}_{i}\right\}=2 h_{y_{i}}^{2}
\end{aligned}
$$

where $M\{\cdot\}$ and $D\{\cdot\}$ are symbols of mean and variance respectively; $h_{x_{i}}^{2}=\sigma_{x_{i}}^{2} \frac{E_{i}}{N_{0} P^{2}} ; h_{y_{i}}^{2}=\sigma_{y_{i}}^{2} \frac{E_{i}}{N_{0} P^{2}}$. Here it is taken into account that for the frequency diversity case there are $P$ out of $Q$ virtual branches and the transmitted power has to be divided between them [24].

From (12) and (13) it follows that for both hypothesis the PDF $W(z)$ is always a non-central chi-square distribution.

For analytical evaluation the special cases of "high reliability detection" $\forall h_{x_{i}}^{2}, h_{y_{i}}^{2}>1$, are considered. Then for these conditions, from (12) it follows that for $H_{0}, M\left\{V_{i}\right\}$ and $M\left\{\widetilde{V}_{i}\right\}$ are close to zero, while the variances are close to one, then $\left.W(z)\right|_{H_{0}}$ is tending to $\chi_{2 Q}^{2}$ central distribution.

For this case, $P_{f a}$ is well known [23]:

$$
P_{f a} \sim \frac{1}{(Q-1) !} \Gamma\left(\Lambda_{0}, Q\right)=\exp \left(-\Lambda_{0}\right) \sum_{q=0}^{Q-1} \frac{\left(\Lambda_{0}\right)^{q}}{q !}
$$

Fixing the level of $P_{f a}$ one can find $\Lambda_{0}$ and once more, applying the conditions $\forall h_{x_{i}}^{2}, h_{y_{i}}^{2}>1$ from (13) it follows that for the hypothesis $H_{1}$, the variances $D\left\{V_{i}\right\}$ and $D\left\{\widetilde{V}_{i}\right\}$ are going to be extremely large.

In this case $P_{M}$ is:

$$
\begin{gathered}
P_{M} \sim \frac{\Lambda_{0}^{Q}}{Q !} \prod_{i=1}^{Q} \frac{\left(1+\beta_{i}^{2}\right)\left(1+q_{i}^{2}\right)}{2 \bar{h}_{i}^{2} \beta_{i}} \\
\cdot \exp \left[-\frac{q_{i}^{2}\left(1+\beta_{i}^{2}\right)}{2 \beta_{i}^{2}}\left(\cos ^{2} \varphi_{0_{i}}+\beta_{i}^{2} \sin ^{2} \varphi_{0_{i}}\right)\right]
\end{gathered}
$$

where $\bar{h}_{i}^{2}=\frac{2 E_{i}}{N_{0} P^{2}}\left(\sigma_{x_{i}}^{2}+\sigma_{y_{i}}^{2}+m_{x_{i}}^{2}+m_{y_{i}}^{2}\right)$; the remaining parameters were introduced at (3). For one sided- Gaussian distribution (15) becomes:

$$
P_{M}=\frac{\Gamma(Q+1) \Lambda_{0}^{Q}}{2 \bar{h}_{i}^{2 Q} Q ! \Gamma\left(\frac{Q}{2}\right) \sqrt{\pi}}
$$

Now let us repeat the same analysis as before, but for Nakagami fading channels (see Section 5). Assuming non-correlated homogeneous conditions for the fading in all "virtual branches" one can get:

$$
P_{M}=\frac{\Lambda_{0}^{Q}}{Q !}\left(\frac{2 m}{2 m+\bar{h}^{2}}\right)^{Q m}
$$

where $\Lambda_{0}$ can be found from (14) and " $m$ " from (4).

\subsection{Totally Dependent Virtual Branches (Flat Fading) in GG Channel}

In this case the fading processes at all the virtual branches are totally correlated. Obviously it means that the resulting SNR after combining is: $\bar{h}_{\Sigma}^{2}=\frac{1}{P^{2}} \sum_{i=1}^{Q} \bar{h}_{i}^{2}$; thus the problem can be transferred to the quadrature addition algorithm for one equivalent branch, i.e. without diversity but, with the GG model of flat fading:

$$
V_{o}^{2}+\widetilde{V}_{o}^{2} \geq \Lambda_{0}
$$

where $V_{0}^{2}=\sum_{i=1}^{Q} V_{i}^{2}, \widetilde{V}_{0}^{2}=\sum_{i=1}^{Q} \widetilde{V}_{i}^{2}$. Here formulas (12)

and (13) are valid but for conditions of single channel, i.e., without index " $i$ ".

Then $Q_{\text {eqv }} \equiv 1$ and from (14)

$$
\begin{aligned}
& P_{f a}= \exp \left(-\Lambda_{0}\right), \Lambda_{0}=\ln \frac{1}{P_{f a}} \text { and } \\
& P_{M}=\frac{\ln \frac{1}{P_{f a}}\left(1+\beta^{2}\right)\left(1+q^{2}\right)}{2 \beta \bar{h}_{i}^{2} \exp \left[\frac{q_{i}^{2}\left(1+\beta^{2}\right)}{2 \beta^{2}}\left(\cos ^{2} \phi_{0}+\beta^{2} \sin ^{2} \phi_{0}\right)\right]}
\end{aligned}
$$

Dependence between $P_{f a}$ and $P_{M}$ is usually called as "Receiver Operational Characteristic-ROC" and they are presented at Figures 1-3, where the continuous lines correspond to case $a$ and the dotted lines to case $b$.

Comparison of (19) and (15) deserves some comments.

1) When in both scenarios $\forall h_{i}^{2}$ are equal and $P_{f a}$ is fixed, then $P_{M}$ from (19) is much more than $P_{M}$ from (15). The latter can be explained by the diversity effect at (19), see also [8,25].

2) Then it is reasonable to choose a small set of delays and multiple frequencies $(Q \leq 5[10,25])$ in order to provide (if the channel conditions allow it) statistically inde- 


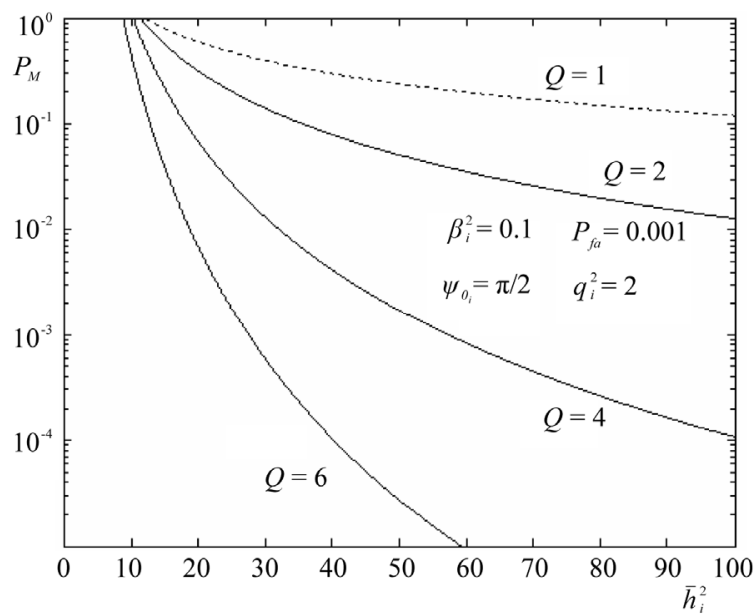

Figure 1. ROC, continuous (15), dotted (19).

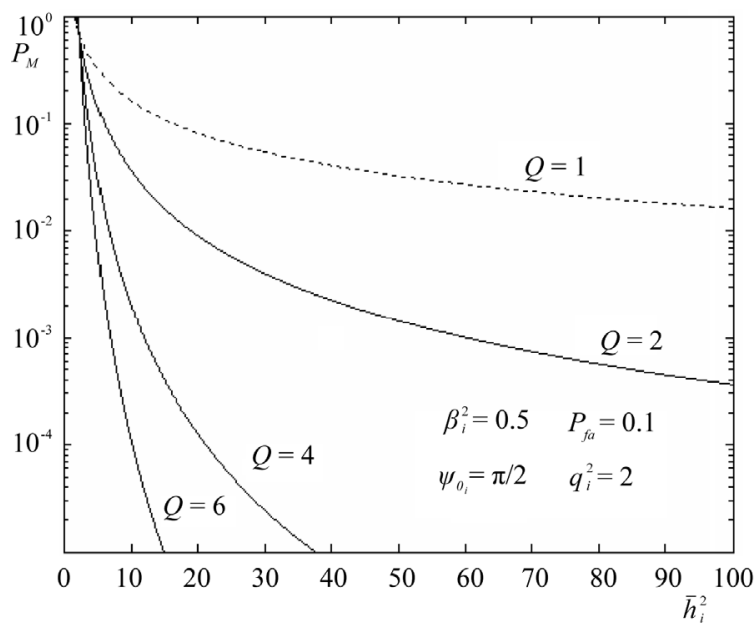

Figure 2. ROC (15) and (19) for another set of parameters.

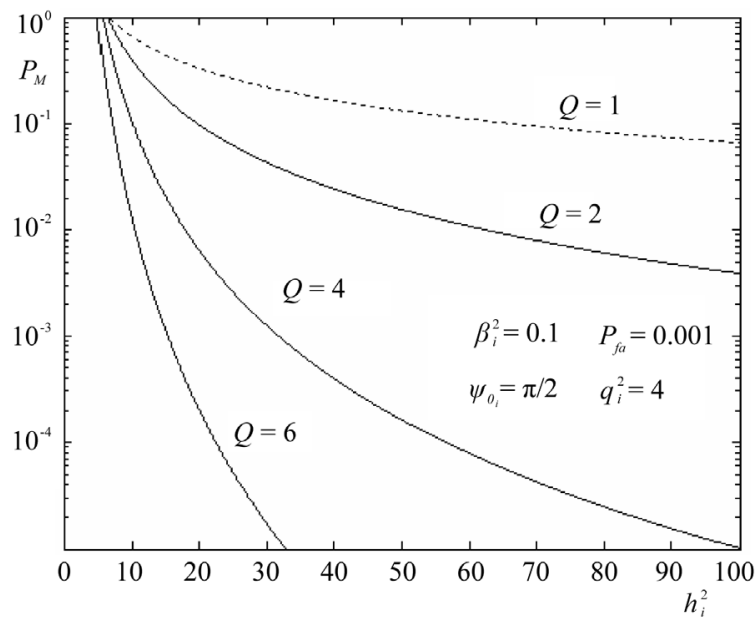

Figure 3. ROC (15) and (19) for a different set of parameters.

pendent fading in those virtual branches, i.e. it is reasonable to "sacrifice" the numbers of $P$ and $N$ by bigger intervals between $\{\alpha\}$ and $\{\tau\}$ so as to artifi- cially create independent fading in the frequency and delay domains, which certainly leads to better noise immunity after "diversity combining". So, appropriate choice of cyclostationary features $Q=N P$ of the desired signals of PU can significantly improve their ROC properties.

\subsection{One Special Case of Covariance Matrix for Correlated Branches at Quadratic Incohe- rent Addition Algorithm}

Let us consider in the following one special case of the covariance matrix for quadrature components $x=\left\{x_{l}\right\}_{1}^{Q}$ and $y=\left\{y_{l}\right\}_{1}^{Q}$ : assume that across the branches all $x_{l}$ or $y_{l}$ Gaussian components are correlated with coefficients $R_{x}$ or $R_{y}$ and there is no cross-correlation at all between $x_{l}$ and $y_{l}$ Gaussian components. One can see, that this assumption restricts (in general) the type of the covariance matrix of the GG channel model but might be useful for the first step examination of the influence of the covariance between virtual (but not only virtual!) branches at the noise immunity characteristics of the SU: consider, for example, SU which applies multi-antenna receiving system, etc.

It is well known that for each pair of $x$ or $y$ Gaussian variables, by the well known angle rotation linear transform it is possible to obtain a new set of statistically independent Gaussian variables: rotating of the coordinate system (linear transform) by the angle

$\varphi=\arctan \left\{2 R \frac{\sigma_{1} \sigma_{2}}{\sigma_{1}^{2}-\sigma_{2}^{2}}\right\}$, where $R$ is a correlation coefficient; $\sigma_{1}^{2}, \sigma_{2}^{2}$-are variances of two correlated Gaussian quadrature components, while new Gaussian variables are statistically independent $[17,11]$.

In order to provide tractable analytical results, in the following only the case $Q=2$ for the algorithm (11) will be considered.

Then noise immunity analysis can be done in the same way as it was done at III $a$, but the means and variances for hypothesis $H_{0}$ and $H_{1}$ have to be calculated by the formulas:

$$
\sigma_{I, I I}^{2}=\frac{2 \sigma_{1}^{2} \sigma_{2}^{2}\left(1-R^{2}\right)}{\left(\sigma_{1}^{2}+\sigma_{2}^{2}\right)\left[1 \pm \sqrt{1-\left(1-R^{2}\right) \frac{4 \sigma_{1}^{2} \sigma_{2}^{2}}{\sigma_{1}^{2}+\sigma_{2}^{2}}}\right]}
$$

where $\sigma_{1}^{2}, \sigma_{2}^{2}$ correspond to the variances of the quadrature components, calculated for different hypothesis $H_{0}$ and $H_{1}$ (see (12), (13)); $\sigma_{I, I I}^{2}$ are new variances of the quadrature components after angle rotation (for each two branches).

Moreover, assuming in the following for simplicity $R_{x}$ $=R_{y}=R$, in the same way as before one gets: 


$$
\begin{aligned}
& m_{I}=\left(m_{1} \cos \psi-m_{2} \sin \psi\right) \\
& m_{I I}=\left(m_{1} \sin \psi-m_{2} \cos \psi\right)
\end{aligned}
$$

where $m_{1}, m_{2}$ are expectations of the initial quadrature components (see also (12),(13) ); $m_{I}, m_{I I}$ are new means after angle rotation.

Now all the set of these parameters can be considered as new parameters of the GG model with the statistically independent branches. So the noise immunity (ROC) can be calculated in the same way as in III $a$ (see formulas (14), (15)). This calculation, (in general) is rather cumbersome, because the new parameters of the GG model come from rather complex expressions (see above).

Therefore assuming that $P_{f a}$ and $P_{M}$. are much less than one as it was done earlier, it is possible to apply the asymptotic calculus (see $[10,11]$ ).

Particularly, for the hypothesis $H_{0}$ (see III $a$ ) all means will be close to zero and all variances will be equal to $\left(1-R^{2}\right)$. So $P_{f a}$ can be calculated by (14) but with the new threshold $\Lambda^{\prime}=\Lambda_{0} /\left(1-R^{2}\right)$, depending on $R$, but the method of calculus is the same as in IIIa.

Then for the asymptotic case $P_{M}<<1$, one can get:

$$
\begin{aligned}
& P_{M} \sim \frac{\Lambda^{\prime 2}}{2} \exp \left\{-\frac{m_{I_{x}}^{2}}{2\left(1+\sigma_{I_{x}}^{2}\right)}-\frac{m_{I I_{x}}^{2}}{2\left(1+\sigma_{I I_{x}}^{2}\right)}-\frac{m_{I_{y}}^{2}}{2\left(1+\sigma_{I_{y}}^{2}\right)}\right. \\
& \left.-\frac{m_{I I_{y}}^{2}}{2\left(1+\sigma_{I I_{y}}^{2}\right)}\right\}\left[1+\frac{\sigma_{I_{x}}^{2}}{2\left(1+\sigma_{I_{x}}^{2}\right)}\left(1+\frac{m_{I_{x}}^{2}}{\sigma_{I_{x}}^{2}\left(1+\sigma_{I_{x}}^{2}\right)}\right)+\right. \\
& \frac{\sigma_{I I_{x}}^{2}}{2\left(1+\sigma_{I I_{x}}^{2}\right)}\left(1+\frac{m_{I I_{x}}^{2}}{\sigma_{I I_{x}}^{2}\left(1+\sigma_{I I_{x}}^{2}\right)}\right)+\frac{\sigma_{I_{y}}^{2}}{2\left(1+\sigma_{I_{y}}^{2}\right)}\left(1+\frac{m_{I_{y}}^{2}}{\sigma_{I_{y}}^{2}\left(1+\sigma_{I_{y}}^{2}\right)}\right) \\
& \left.+\frac{\sigma_{I I_{y}}^{2}}{2\left(1+\sigma_{I I_{y}}^{2}\right)}\left(1+\frac{m_{I I_{y}}^{2}}{\sigma_{I I_{y}}^{2}\left(1+\sigma_{I I_{y}}^{2}\right)}\right)\right] .
\end{aligned}
$$

This formula is for the GG channel model and is rather general in the sense that it does not provide a "transparent picture" about the dependence of $P_{M}$, for example, on $R$ etc; it requires implicitly numerical calculus.

Let us consider a special case: $\sigma_{I_{x}}^{2}=\sigma_{I_{X}}^{2}=\sigma_{I_{y}}^{2}=$ $\sigma_{I I_{y}}^{2}=\sigma^{2}$; next, introducing $\gamma_{0_{I}}^{2}=m_{I_{x}}^{2}+m_{I_{y}}^{2}$ and $\gamma_{0_{I I}}^{2}=m_{I I_{x}}^{2}+m_{I I_{y}}^{2}$ one can get:

$$
P_{M} \sim \frac{\Lambda^{\prime 2}}{2 \sigma^{4}} \exp \left\{-\frac{\gamma_{0_{I}}^{2}}{2 \sigma^{2}}-\frac{\gamma_{0_{I I}}^{2}}{2 \sigma^{2}}\right\}\left(3+\frac{\gamma_{0_{I}}^{2}}{2 \sigma^{4}}+\frac{\gamma_{0_{I I}}^{2}}{2 \sigma^{4}}\right) .
$$

For this special case it can be found that:

$$
\sigma^{2}=\frac{2 h_{0_{1}}^{2} h_{0_{2}}^{2}\left(1-R^{2}\right)}{\left(h_{0_{1}}^{2}+h_{0_{2}}^{2}\right)\left[1 \pm \sqrt{1-\left(1-R^{2}\right) \frac{4 h_{0_{1}}^{2} h_{0_{2}}^{2}}{\left(h_{0_{1}}^{2}+h_{0_{2}}^{2}\right)^{2}}}\right]}
$$

when $\frac{\gamma_{0_{I}}^{2}}{2 \sigma^{2}}$ and $\frac{\gamma_{0_{I I}}^{2}}{2 \sigma^{2}}$ are much less than one (strong fading), then:

$$
\begin{aligned}
& P_{M} \sim \frac{3 \Lambda^{\prime 2}}{2 \sigma^{4}}=\frac{3}{2}\left(\Lambda^{\prime}\right)^{2} \frac{1}{h_{0_{1}}^{2} h_{0_{2}}^{2}\left(1-R^{2}\right)} \\
& P_{M} \sim \frac{3 \Lambda^{\prime 2}}{2 \sigma^{4}}=\frac{3}{2}\left(\Lambda^{\prime}\right)^{2} \frac{1}{h_{0_{1}}^{2} h_{0_{2}}^{2}\left(1-R^{2}\right)}
\end{aligned}
$$

The last formula shows that, losses related to correlation between diversity branches depend mainly on $\frac{1}{\left(1-R^{2}\right)}$; this result was in some sense predictable (see $[7$, 8] for example).

When the fading follows the truncated Gaussian PDF then: $P_{M} \sim \frac{\Lambda^{\prime 2}}{2} \frac{1}{\sqrt{\left(1+\sigma_{I_{y}}^{2}\right)\left(1+\sigma_{I I_{y}}^{2}\right)}}\left(\frac{3}{2}+\frac{\sigma_{I_{y}}^{2}}{2\left(1+\sigma_{I_{y}}^{2}\right)}+\frac{\sigma_{I I_{y}}^{2}}{2\left(1+\sigma_{I I_{y}}^{2}\right)}\right)$ when $P_{M} \ll 1$, then:

$$
P_{M} \sim \frac{\left(\Lambda^{\prime}\right)^{2}}{2} \frac{5}{8 h_{0_{1}}^{2} h_{0_{2}}^{2}\left(1-R^{2}\right)}
$$

One can see that losses once more depend on $\frac{1}{\left(1-R^{2}\right)}$ as well.

The same character of losses can be found for significantly Rician character of the GG model; so, it can be considered as a rather "universal" dependence of losses on the correlation coefficient value.

Of course changes of the threshold, which depends on " $R$ ", influence the character of the dependence of ROC on the correlation properties of the GG model in a nonlinear way, but this will be discussed elsewhere in the future.

Concluding the material of this section, it is worth to mention, that from the theory of diversity combining it is well known $[7,8]$ that correlation between branches has influence, mainly, on the noise immunity characteristics (ROC, in our case) when resulting SNR is rather high, i.e. $P_{M}$ is much less than one.

\section{Suboptimal Algorithms and Their Noise Immunity}

The first suboptimal algorithm considered hereafter will be an energetic receiver where the desired signal is represented in the way: $x(t)=\sum_{i=1}^{B} \alpha_{i} \varphi_{i}(t)$; here $\left\{\varphi_{i}(t)\right\}_{1}^{B}$-are orthonormal functions.

According to $[22,23]$ the corresponding algorithm (NPT) can be represented in the way: 


$$
\begin{gathered}
H_{0}: \sum_{j=1}^{B}\left[\int_{0}^{T} z(t) \phi_{i}(t) d t\right]^{2}=n(t) \\
H_{1}: \sum_{j=1}^{B}\left[\int_{0}^{T} z(t) \phi_{i}(t) d t\right]^{2}, \text { i.e. }=\hat{r}_{x x^{*}}+n(t),
\end{gathered}
$$

where $B$ is the number of orthonormal functions $\left\{\varphi_{i}(t)\right\}_{1}^{B}$ applied for the expansion of the desired signal $x(t)$; the received signal is $z(t)=x(t)+n(t), T$ is the time of analysis.

Now, for the representation of $x(t)$ and $n(t)$ let us apply the F-basis in the same way as it was done at [10], (see also [22] and references therein).

Then:

$$
\begin{aligned}
& x(t)=\sum_{k=0}^{B} a_{k} \cos \left(k \omega_{0} t\right)+b_{k} \sin \left(k \omega_{0} t\right) \\
& n(t)=\sum_{k=0}^{B} \alpha_{k} \cos \left(k \omega_{0} t\right)+\beta_{k} \sin \left(k \omega_{0} t\right)
\end{aligned}
$$

where $\omega_{0}=2 \pi / T, B=2 F T, F=\frac{k_{2}-k_{1}}{F}+1-$ frequency bandwidth, $k_{2}, k_{1}$ are upper and lower indexes taken into account here for the F-series expansion.

Then:

$$
\begin{aligned}
& H_{0}: \frac{1}{2} \sum_{k=0}^{B}\left(\alpha_{k}^{2}+\beta_{k}^{2}\right)=n(t) \\
& H_{1}: \frac{1}{2} \sum_{k=0}^{B}\left(\alpha_{k}+a_{k}\right)^{2}+\left(\beta_{k}+b_{k}\right)^{2} \geq \Lambda_{0}
\end{aligned}
$$

As all $\left\{a_{k}\right\},\left\{b_{k}\right\},\left\{\alpha_{k}\right\}$ and $\left\{\beta_{k}\right\}$ are Gaussian distributed coefficients, the left side in (22) has central or non-central $\chi_{2 B}^{2}$ distributions respectively. Defining those left sides in (22) as $\lambda_{1}$ and $\lambda_{2}$ (see $[10,22]$ and Section 4 one has to apply:

$$
\begin{aligned}
& W\left(\lambda_{1}\right)=\frac{1}{D 2^{B / 2} \Gamma(B / 2)}\left(\frac{\lambda_{1}}{D}\right)^{B / 2-1} \exp \left(-\frac{\lambda_{1}}{2 D}\right) \\
& W\left(\lambda_{2}\right)=\frac{1}{2 D}\left(\frac{\lambda_{2}}{\Delta}\right)^{\frac{B / 2-1}{2}} \exp \left(-\frac{\lambda_{2}+\Delta}{2 D}\right) I_{B / 2-1}\left(\frac{\sqrt{\lambda_{2} \Delta}}{D}\right)
\end{aligned}
$$

where $\Delta=\sum_{k=0}^{B} a_{k}^{2}+b_{k}^{2}=2 P_{x}$ is the average power of $x(t)$ and the parameter $D=\frac{N_{0} T}{2}$.

Then the threshold $\Lambda_{0}$ can be easily found from (14) where $Q \triangleq B$ and $P_{f a}$ are fixed.

The detection probability $P_{M}$ is:

$$
P_{M}=\frac{1}{2} \exp \left(-\frac{h^{2}}{2}\right) F\left(\frac{\Lambda_{0}}{D}, h^{2}, B\right) \leq \frac{1}{2} \exp \left(-\frac{h^{2}}{2}\right)
$$

where $0 \leq F\left(\frac{\Lambda_{0}}{D}, h^{2}, B\right) \leq 1$ is the Cumulative Distribution Function (CDF) of the non-central $\chi_{2 B}^{2}$ PDF.

The upper bound of $P_{M}$ for the GG channel model with flat fading is known from [8]:

$$
\begin{gathered}
P_{M} \leq \frac{1}{C_{1}} \exp \left\{-\frac{q^{2} h^{2}}{2\left(1+q^{2}\right)}\left[\frac{\left(1+\beta^{2}\right)\left(1+q^{2}\right) \cos ^{2} \phi_{0}}{\left(1+\beta^{2} \bar{h}^{2}\right)}\right.\right. \\
\left.\left.+\frac{\left(1+\beta^{2}\right)\left(1+q^{2}\right) \sin ^{2} \phi_{0}}{\left(1+\bar{h}^{2}\right)}\right]\right\}
\end{gathered}
$$

where $\bar{h}^{2}=\left\langle\gamma^{2}\right\rangle \frac{2 E}{P^{2} N_{0}}$,

and $C_{1}=2 \sqrt{1+\frac{\beta^{2} \bar{h}^{2}}{\left(1+\beta^{2}\right)\left(1+q^{2}\right)}}$

An exact tractable analytical expression of $P_{M}$ for the GG model is not available.

In absence of fading it is possible to obtain an analytical result in the following way.

First, representing the Bessel function as in [18] in the way:

$$
I_{B / 2-1}\left(\sqrt{h^{2} y}\right)=\sum_{k=0}^{\infty} \frac{\left(h^{2} y\right)^{\frac{B / 2-1}{2}+k}}{k ! \Gamma(B / 2+k)},
$$

then the $P_{M}$ from (24) is:

$$
P_{M}=\frac{1}{2} \exp \left(-\frac{h^{2}}{2}\right) \sum_{k=0}^{\infty} \frac{\gamma\left(B / 2+k, \frac{\Lambda_{0}}{2 D}\right)\left(\bar{h}^{2}\right)^{\frac{B}{4}-\frac{1}{2}+k}}{k ! \Gamma(B / 2+k) 2^{k-1}}
$$

where $B=2 F \tau, \gamma(\alpha, x)$ - is the lower incomplete gamma function.

Analysis of (27) shows that influence of $B$ can be significant and it can be shown that for fixed $P_{f a}$ or $\Lambda_{0} / D$, while $B$ grows, $P_{M}$ also grows. To the best of our knowledge, influence of $B$ and not only of $\bar{h}^{2}$ on the noise immunity of the energetic (autocovariance) receiver was first stressed in [22].

Then for the multiple cyclic frequency case, when the number of frequencies $P$ is rather large while $T$ is fixed, $F$ is large as well and $P_{M}$ grows.

Therefore the energetic detector is not definitely a good candidate for spectrum sensing for this scenario, as its $P_{M}$ is much worse than for the optimum detector (see the previous section). In some sense this comment coincides with the simulations [4], besides that there the energetic 
detection was not implemented in the same way as mentioned above.

Another option for suboptimal detection is to take advantage of the analogy between multiple cyclic frequency detection and quadratic diversity combining and apply a suboptimal variant of incoherent diversity addition (see [7, 8]). Hereafter a selection (switching) combining method was chosen, assuming that fading has a Nakagami PDF (see [4]). See formula (4) to adjust parameters of Nakagami PDF and four-parameter distribution.

There are several different approaches for switching combining but in the following we will analyze only the algorithm of selection of the "virtual branch" with $\gamma^{\prime}=$ $\max _{\gamma} \gamma_{i}, \quad i=\overline{1, Q_{0}}$.

Let us assume here, for simplicity, the homogeneous fading conditions then, the distribution of the maximum value of the identically distributed random values is [11]:

$$
W\left(\gamma^{\prime}\right)=Q W\left(\gamma=\gamma^{\prime}\right)\left[\int_{0}^{\gamma^{\prime}} W(y) d y\right]^{Q-1}
$$

$$
\text { If } W(\gamma) \text { is [19]: }
$$

$$
W(\gamma)=\frac{2 m^{m} y^{2 m-1}}{\Gamma(m) \Omega^{2 m}} \exp \left(-m \frac{y^{2}}{\Omega^{2}}\right)
$$

Then it is necessary to average $P_{M}$ for one virtual branch without fading through (28) with the help of (29) while $P_{f a}$ is:

$$
P_{f a}=\exp (-\Lambda)
$$

where $\Lambda$ is a threshold.

The $P_{M}$ of the channel without fading is:

$$
P_{M}=Q(\sqrt{h}, \Lambda)
$$

Following the above mentioned procedure for the case $Q=2$ and $h>1$ one can get from (28)-(31) an approximate formula:

$$
P_{M}=\frac{\Lambda^{2}}{\left(1+\frac{h^{2}}{2 m}\right)^{m}}\left\{1-\sum_{i=0}^{m-1} \frac{(m-1+i) !\left(1+\frac{h^{2}}{2 m}\right)^{m}}{i !(m-1) !\left(2+\frac{h^{2}}{2 m}\right)^{m+i}}\right\}
$$

for $m$-integers.

One can compare this method of switching combining (with fixed $\bar{h}^{2}$ and $Q=2$ ) with the optimum approach (see (14), (17)).

Please notice that in fading channel conditions when the number of virtual branches is growing, one encounters the so-called "hardening effect", i.e. while $Q$ is increasing, the increment of noise immunity might be low.

Therefore, with $Q=2$ there is a good option to compare the effectiveness of the selection combining method with the optimum one.

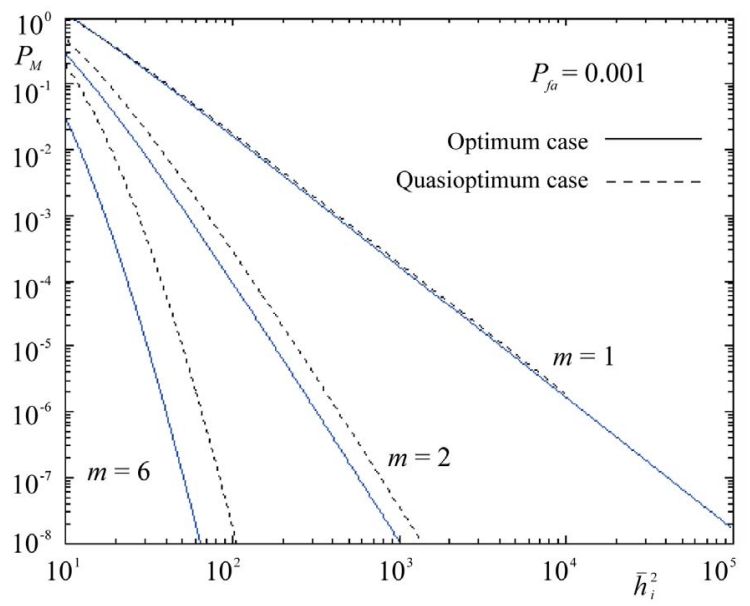

Figure 4. Comparison of the ROC for the optimum (17), and quasioptimum (32), cases.

In Figure 4 the ROC for this method is presented, where for comparison some of the "optimum" ROC's, see (17), are presented. One can see that the energetic losses for $P_{M}=10^{-4}$ are rather small and for $m=1$ are negligible.

In the same manner as above, the well known set of sub-optimum combining algorithms can be applied: other methods of switching combining, linear (weighted and non-weighted) addition, etc. Their application is rather straightforward and is not presented here.

Some discussion regarding the obtained results

One can ask: if both algorithms (11) and (12) rely on quadratic addition of the F-coefficients, then why their noise immunity is so different, particularly with the GG channel fading? What is going on? The answer is rather straightforward.

At (11) the object of the quadratic addition are the Fcoefficients, but from the autocovariance function of the output of the multiple cyclic frequency optimum detector, i.e. after optimum processing of the quadrature components of the input signals. It is also possible to provide statistically independent fading of the virtual branches for incoherent addition by properly choosing the cyclic frequencies and delays, etc. which drastically increase the noise immunity (through the diversity effect).

In contrary, the energetic receiver, as it is in (20)-(22), does not apply specific properties of the cyclic frequencies and just extracts the total energy of the aggregate input signal. It is often hardly possible for this case to the F-coefficients of the input signal to exhibit statistical independency in fading conditions.

Moreover, for the energetic receiver (22) the noise immunity, even in the case of a constant channel (without fading), goes down while the bandwidth $F$ grows $(B=2$ $F T$, with $T$ fixed) as the noise power grows. Therefore the energetic receiver for multiple cyclic frequency signals might be useless when $F T \gg 1$. 
For a quasi-optimum alternative for optimum quadratic combining it is possible to consider all the set of switching combining algorithms (see (30) for example), as well as a whole set of quasi-optimum algorithms of non-coherent diversity combining such as a set of linear combining methods with rather low energetic losses for the fixed $P_{f a}$ and $P_{M}$.

\section{Collaborative Spectrum Sensing with Censoring}

Here as in previous section it is supposed that spectrum sensing is based on the cyclostationary properties of the signals of the primary users (PU) and the secondary users (SU) are spatially distributed within certain area. All the set of SU can sense the whole frequency band of interest, or each SU may sense just a partial band. Hereafter it will be assumed that all SU are sensing the same frequency band. In both cases of spectrum monitoring, SU have to share the sensing information between them or might be coordinated by a Fusion Center.

It seems reasonable that, no matter what kind of exchange is used, the local decision information has to be obtained by a minimum set of observations $\mathrm{M}$ in (5), while $\mathrm{N}$ and $\mathrm{P}$ are fixed ${ }^{5}$. In other words, the time of analysis in (5) has to be reduced as much as possible; meanwhile the amount of transmitted data has to be reduced as well.

Then it is opportunistic to apply the sequential analysis of A. Wald [26] where ML test, in contrary to NPT, has to be compared with two thresholds related to requirements of $P_{f a}$ and $P_{M}$.

Let us suppose that for the latter, highly reliable final results for the test are predefined, so $P_{f a}$ and $\mathrm{PM}$ have to be rather low. This might be a rational way to make censoring for the local test as only reliable information has to be forwarded to the FC or other SU.

One has to notice that each SU will obtain those reliable final results (whether PU exists or not) at different time instants. This information has to be sent to other fellow SU or to FC in binary way.

Next let us consider several rather general but different scenarios of collaborative spectrum sensing.

- Each $n$-th SU, $n=\overline{1, K}$, passes, after time "T", the information of " $z_{n}$ " (not binary) to the system of quadratic addition at the FC. So,

$$
\begin{aligned}
& Z=\sum_{n=1}^{K} z_{n} \quad \text { or } \\
& Z=\sum_{j=1}^{K Q} z_{j}
\end{aligned}
$$

\footnotetext{
${ }^{5}$ Meanwhile one has to notice, that asymptotic conditions for $M(T)$ are assumed to be valid here in order to preserve the uncorrelated conditions for F-coefficients in (5).

${ }^{6}$ Some modifications of the majority diversity addition can be found at $[13,28]$, etc.
}

Then $\mathrm{Z}$ might be analyzed by the NPT or by sequential analysis (see below) assuming hereafter that the channel $\mathrm{SU} \rightarrow \mathrm{FC}$ is error free. So after final addition, the result of quadratic diversity addition of KQ virtual branches (or of K SU) is analyzed, assuming statistically independent fading along all summations (see (10) and (11) above).

This scenario can be called as a distributed optimum incoherent "SIMO passive radar" and its characteristics are equal to (14), (15) with the number of virtual branches KQ.

- Each of the $n$-th SU make an individual decision regarding to the presence of PU and then send the binary decision to the FC by error free channels. Assuming that all those decisions are statistically independent, the final result at the FC can be obtained according to the majority rule (see for example [27]) with the majority not- weighted (or weighted) diversity addition method. This case can be also called "SIMO radar" but in contrary to the first one it is non-optimum. In the following the topics related to those issues will be thoroughly considered.

Majority diversity addition and weighted majority addition (WMA) in collaborative spectrum sensing

If the majority principle is applied at FC, then the decision is made by analysis of the partial decisions at each SU (here SU acts as a "virtual" diversity branch) and the decision which takes place at the majority of the branches is favored. This method is called "majority diversity addition"

If partial solutions are binary and the number of virtual branches is odd, there cannot be any collision in the final decisions for such method.

Let $\mathrm{K}=2 q-1$ and " $\mathrm{P}_{0}$ " denote the existence of PU after " $q$ " tests on the branches.

So if after " $m-1$ " probes on the virtual branches one gets " $q-1$ " results of existence of PU and " $m$-th" probe gives the same, then for the " $q$ " test one gets the probability of this event as

$$
P\left(\mathrm{P}_{0}\right)=C_{m-1}^{q-1} P_{1}^{q}\left(1-P_{1}\right)^{m-q}
$$

The probability of $\mathrm{P}\left(\mathrm{P}_{0}\right)$ is a sum of statistically independent probabilities of probes (34) through all "m" from $m-q$ up to $m=2 q-1$, i.e.:

$$
P\left(\mathrm{P}_{0}\right)=\sum_{m-q}^{2 q-1} C_{m-1}^{q-1} P_{1}^{q}\left(1-P_{1}\right)^{m-q}
$$

where $P_{1}$ can be $P_{f a}$ or $P_{M}$, so $\mathrm{P}\left(\mathrm{P}_{0}\right)$ is a final probability of false alarm or error detection (see for example [11,27] as well), depending which one of hypothesis is considered.

If one defines " $n$ " in the way $n=m-q$, it yields:

$$
P\left(\mathrm{P}_{0}\right)=\sum_{n=0}^{q-1} C_{q+n-1}^{q-1} P_{1}\left(1-P_{1}\right)^{n}
$$


At the same time, from the theory of diversity combining it is known that majority addition is equivalent to the optimal incoherent addition with the number of branches (here virtual ones) "q", i.e., to incoherent (quadratic) addition with almost twice less branches.

So, comparing the characteristics of majority addition with those of the optimum SIMO radar one can see several limitations of the former:

- Optimum SIMO radar with incoherent addition actually operates with almost twice more virtual branches and therefore provides significantly better detection characteristics (ROC's);

- The majority addition operates successfully only with odd number of virtual branches, while optimum SIMO radar operates with any number of branches.

The price one has to pay for the advantages of the optimum SIMO radar is a more complex data transmission scheme: in the majority addition, simply binary results are transmitted, and for SIMO radar the information of the " $z$ " value for each SU has to be transmitted to FC through error free channels.

Is it possible to improve the ROC properties of the majority addition in order to make them approach to those of the optimum SIMO radar?

In order to approach the noise immunity properties of the majority addition to those of the optimum incoherent addition, some modifications of the former were proposed.

One of them, the so-called "weighted majority addition" was proposed at $[29,30]$. The idea of this method is rather simple: introduce in the majority addition algorithm information of the channel gains for each partial solution, or in other words introduce "weights" in the procedure of the majority addition algorithm. In this way the channel gains for the diversity "branches" work as weighting coefficients in the process of majority selection. It was shown that this suboptimal method provides results very close to those of the optimum incoherent addition [29,30] if the communication scenario allows taking advantages of channel gains.

One can see that it is not the case for one of the scenarios at FC: each result of detection at SU was obtained through the optimum quadratic addition by the SU itself, so the resulting fading at SU has a very low variance when $Q$ is rather large (hardening effect) (see, for example $[10,25])$. Therefore, it is hardly possible to improve the results of majority addition by introducing weighting coefficients as all the weights might be practically equal. But it is known that if the channels are sufficiently heterogeneous, the hardening effect does not even appear or it appears very slowly, while $\mathrm{Q} \rightarrow \infty$ at the SU. So, let us consider another extreme special case. Let us assume that

\footnotetext{
${ }^{7}$ In relation to the fading model assumed here for simplicity, see formula (4) for the definition of the parameter $m$ through the parameters of the Generalized Gaussian model.
}

the fading at the SU's are so heterogeneous, that practically all quadrature addition algorithms do not work as the diversity combining algorithm and each $\mathrm{SU}$ have $\mathrm{Q} \approx$ 1 (single reception) with $\mathrm{m}$-distributed fading ${ }^{7}$ and the fading is generally heterogeneous.

With this assumption one can see that the problem is converted to the case of SIMO Radar: SU are sending to the FC binary information of partial decisions together with the information of their weights in order to provide to the FC with weighted addition (the channel SU $\rightarrow$ FC is supposed to be error free).

Let us formulate here an assumption: if the final decisions are taken at $\mathrm{FC}$ by applying the technique of weighted addition of partial decisions, then the SU's have to transmit to the FC not only the information of partial decisions, but information of their reliability as well and all the system (PU, SU, FC) is working as a distributed quasi-optimum SIMO Radar.

Returning back to the above mentioned scenario, one can see that the decision of PU existence in the majority of "branches" can be obtained by the algorithm:

$$
\left.\sum_{j=q+1}^{K} \mu_{j}\right|_{H_{1}}>\left.\sum_{j=1}^{q} \mu_{j}\right|_{H_{0}},
$$

where $\left\{\mu_{j}\right\}_{1}^{K}$ - are magnitudes of the channel gains; hypothesis $H_{1}$ and $H_{0}$ have the same sense as in (10).

From Bayes theorem each of the summands in (37) have the following PDFs:

$$
\begin{aligned}
W\left(\left.\mu_{j}\right|_{H_{0}}\right) & =\frac{\left.W\left(\mu_{j}\right) P_{f a}\right|_{\mu_{j}}}{P_{f a}} \\
W\left(\left.\mu_{j}\right|_{H_{1}}\right) & =\frac{\left.W\left(\mu_{j}\right)\left(1-P_{d}\right)\right|_{\mu_{j}}}{1-P_{d}}
\end{aligned}
$$

and the error $P_{M}$ after addition, finally will take place if the sign of the inequality in (37) changes to the opposite one. Conditions for false alarm are defined in a similar way when PU really does not exist in observations at SU. Let us assume that [19]:

$$
W\left(\mu_{j}\right)=\frac{2 m_{j}^{m j} \mu_{j}^{2 m_{j}-1}}{\Gamma\left(m_{j}\right)\left(\begin{array}{l}
-2 \\
\gamma_{i}
\end{array}\right)^{m_{j}}} \exp \left(-m_{j} \frac{\mu_{j}^{2}}{\left(\begin{array}{l}
-2 \\
\gamma_{i}
\end{array}\right)}\right)
$$

where $m_{j}$ and the corresponding parameters for fourparametric distribution are related by $m_{j} \geq \frac{1}{2}$.

Then introducing the new variable

$$
x_{j}=\mu_{j} \frac{\sqrt{m_{j}+\frac{h^{2}}{2}}}{m_{j}}
$$


one can get:

$$
\begin{aligned}
W\left(\left.x_{j}\right|_{H_{0}}\right) & =\frac{2 m_{j}^{m j} x_{j}^{2 m_{j}-1}}{\Gamma\left(m_{j}\right)\left(\begin{array}{l}
-2 \\
\gamma_{i}
\end{array}\right)^{m_{j}}} \exp \left(-m_{j} \frac{x_{j}^{2}}{\left(\begin{array}{c}
-2 \\
\gamma_{i}
\end{array}\right)}\right) \\
W\left(\left.x_{j}\right|_{H_{0}}\right) & =\frac{2 m_{j}^{m j} x_{j}^{2 m_{j}-1}}{\Gamma\left(m_{j}\right)\left(\frac{h^{2}}{2 m_{j}} \gamma_{i}^{-2}\right)^{m_{j}}} \exp \left(-m_{j} \frac{x_{j}^{2}}{\frac{h^{2}}{2 m_{j}} \gamma_{i}}\right)
\end{aligned}
$$

where Pfa $\ll 1, P_{M}<1$.

From (40) it can be seen that $W\left(\left.x_{j}\right|_{H_{0}}\right)$ and $W\left(\left.x_{j}\right|_{H_{1}}\right)$ have the Nakagami PDF form.

Getting back to (37) and introducing the variables $[29,30]$ :

$$
\begin{aligned}
& \zeta_{q}=\left.\sum_{j=1}^{q} x_{j}\right|_{H_{0}} \\
& \eta_{Q-q-1}=\left.\sum_{j=q+1}^{Q} x_{j}\right|_{H_{1}}
\end{aligned}
$$

one can formally calculate the error probability in (37).

In the general case of the heterogeneous scenarios, according to [31] it is possible to find distributions of $\xi_{q}$ and $\eta_{Q-q-1}$ in a Nakagami PDF form after rather cumbersome calculus. In the most tractable way, according to (77)-(89) in [19], it is possible to provide the error analysis for the following special case, when ${ }^{8}$ :

$$
\frac{\gamma_{1}}{m_{1}}=\cdots \frac{\gamma_{n}}{m_{n}}
$$

here $n$ is the number of Nakagami variables at (41).

Then the sums in (41) will have equivalent Nakagami parameters $0 m \cong m n$ and $0 \gamma^{-2} \cong n \gamma^{-2}$.

Note, that for the general case [31], the calculus of ${ }_{0} \mathrm{~m}$ and ${ }^{-2} \gamma$ can be done mainly numerically. Then from (37) is possible to get (see [30]) for the conditional error probability (with $q$ fixed):

$$
P(q)=\frac{q^{0 m(K-q)} \Gamma\left({ }_{0} m K\right)}{\Gamma\left({ }_{0} m(K-q)+1\right) \Gamma\left({ }_{0} m K\right)\left[\frac{h^{2}(K-q)}{2_{0} m}\right]^{0 m(K-q)}}
$$

But the number of virtual branches " $q$ " with errors, both for $P_{M}$ and $P_{f a}$, is a random variable with Bernoulli PDF, when the virtual branches have statistically independent fading [29,30].

Then:

${ }^{8}$ Formally the following analysis is valid for the general case [30] as well.

$$
P_{\text {error }}=\sum_{q=1}^{K-1} P_{K}(q) P(q)+P_{K}(K)
$$

where $P_{K}(q)=C_{K}^{q} P_{1}^{q}\left(1-P_{1}\right)^{K-q} ; P_{1}-$ is $P_{f a}$ or $P_{M}$ from (14), (15), when $\mathrm{Q}=1$.

Note that at (14), (15) the four parameters have to be previously adjusted by (4) with the value of ${ }_{0} \mathrm{~m}$ and ${ }_{0} \gamma$ at $\stackrel{-2}{h}$.

The formula (43) is "universal" in the sense that the final $P_{M}$ and $P_{f a}$ can be calculated through it, because as it was mentioned above, the inequality of the (37) type can be applied for calculus of false alarm as well.

The ROC's for WMA is presented at Figures 5-7. For comparison purposes we have included the plots corresponding to sections IV $a$ and IV $b$ denoted with continuous lines and with dotted lines the plots corresponding to (43). One can see that for $P_{M}=10-4$ energetic losses are less than $1.5-2 \mathrm{~dB}$.

Finally let us compare the "ideology" of the weighted majority addition with some of the approaches mentioned at [32], see also the references therein, (in [32] it is also assumed an error free channel between SUi $\leftrightarrow$ FC).

The "simple counting" approach [32] is nothing else than selecting for FC decision only "highly weighted" SU. For sure this addition is "less optimum" than the approach in [29] because some of the SU's with small weights do not participate in the decision-taking process at the FC.

Other two methods, namely the Partial Agreement Counting and the Collision Detection, assume the existence of a feedback channel between SU and FC which can be used for comparing partial decisions at the SU and final decisions at the FC in order to select the "true" final decision. This option was not considered in the current analysis.

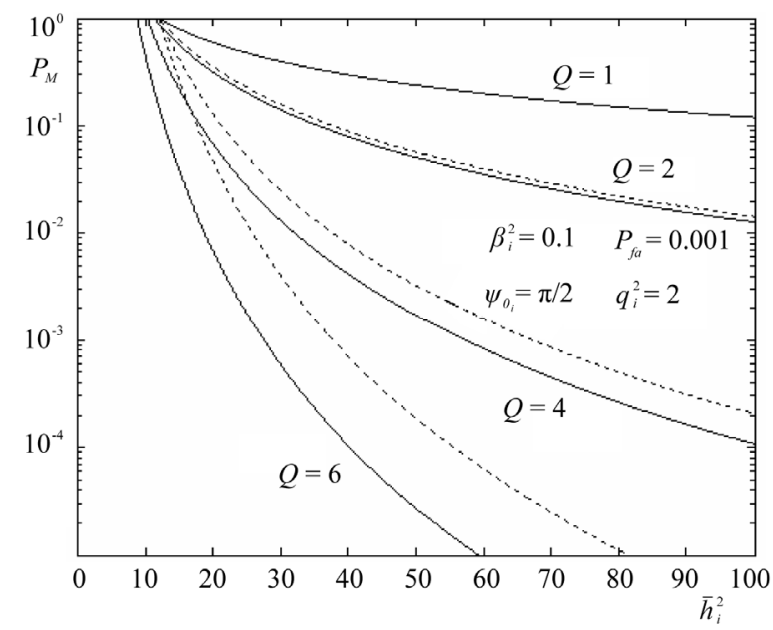

Figure 5. ROC for WMA (15) and (19) - - (43) ---. 


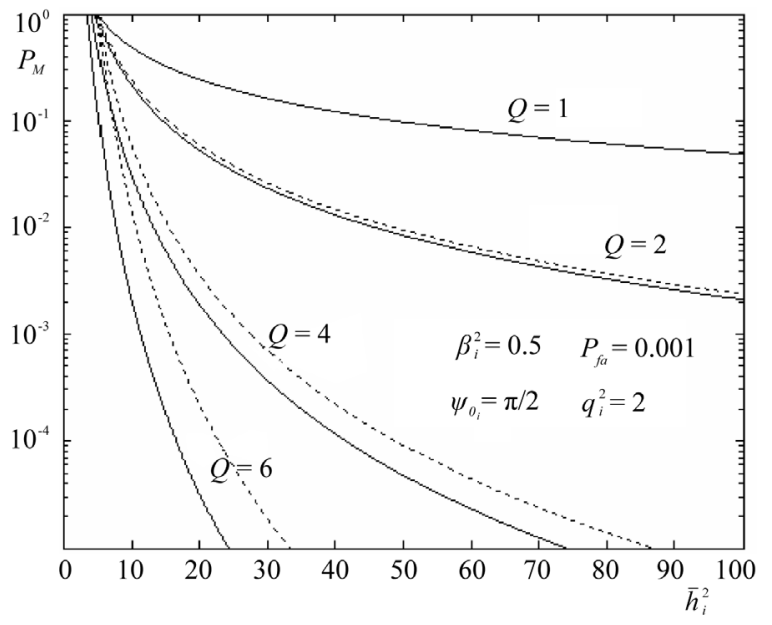

Figure 6. ROC for WMA with different parameters.

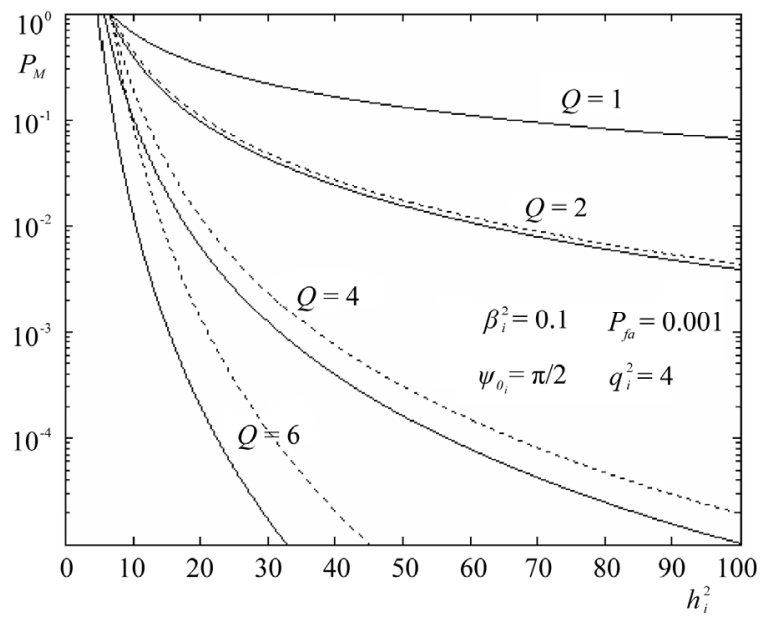

Figure 7. ROC for WMA for different parameters.

For sure, application of the feedback channel opens the possibility to improve the reliability of the final decision at the FC and taking into account that weighted majority addition is a practically optimum incoherent addition, the final characteristics might be better than what it has been mentioned at [32].

\section{Conclusions}

In this paper we have shown that cyclostationary spectrum sensing as well as collaborative spectrum sensing for Cognitive Radio networks can be interpreted as a special case of the concept of optimum or sub-optimum incoherent diversity combining approach (SIMO radar).

It was shown, that as sub-optimum algorithms for this purpose it is possible to apply the whole "gamma" of well known algorithms such as all types of switching combining, as well as linear combining and counting rules (discrete addition), etc.
The concrete detection algorithms (distributed or not) utilizing NPT or sequential tests leads to the so-called SIMO radar algorithms and their ROC's were analyzed here for GG channel fading models in the most general way.

It is worth mentioning here that, application of the cyclostationary properties of the PU signals (through the estimation of the F-coefficients of the autocovariance function) is a convenient but obviously not the unique approach that allows construction of statistically independent virtual diversity branches for the spectrum sensing detection algorithms. For example, for the broad band GG communication channels, virtual branches can be constructed through channel orthogonalizations in the frequency and time domains (in the same way as it was done in [33]), or by choosing statistically independent fading sub-carriers of OFDMA signals (see [34]), etc.

Other emerging problems, such as detailed analysis for correlated virtual branches of the sensing algorithms, adaptive methods of sensing for unknown parameters of GG channels, application of the ideas of the feedback algorithms for collaborative sensing, etc. will be presented by the authors elsewhere.

\section{Acknowledgements}

With the material presented above, the authors would like to acknowledge the outstanding contribution of Prof. D. D. Klovsky, who survived to the Holocaust and passed away almost ten years ago, to the theory of diversity combining in fading channels which as it was shown above is fully operating for the new challenges in communications.

\section{References}

[1] S. Haykin, "Cognitive Radio: Brain Empowered Wireless Communication," IEEE Journal on Selected Areas in Communications, Vol. 23, No. 2, February 2005, pp. 201220. doi:10.1109/JSAC.2004.839380

[2] X. Kang, Y.-C. Liang, H. Krishna and L. Zhang, "Sensing- Based Spectrum Sharing in Cognitive Radio Networks," IEEE Transactions on Vehicular Technology, Vol. 58, No. 8, October 2008, pp. 4649-4654. doi:10.1109/TVT.2009. 2018258

[3] R. Zhang, S. Cui and Y.-C. Liang, "On Ergodic Sum Capacity of Fading Cognitive Multiple-Access and Broadcast Channels," IEEE Transactions on Information Theory, Vol. 55, No. 11, November 2009, pp. 5161-5178. doi:10. 1109/TIT.2009.2030449

[4] J. Lunden, V. Keivunen, A. Huttunen and H. Vincent Poor, "Collaborative Cyclostationarity Spectrum Sensing for Cognitive Radio Systems," IEEE Transactions on Signal Processing, Vol. 57, No. 11, November 2009, pp. 41824195. doi:10.1109/TSP.2009.2025152

[5] A. V. Dandawate and G. B. Giannakis, "Statistical Tests for Presence of Cyclostationarity," IEEE Transactions on 
Signal Processing, Vol. 42, No. 9, September 1994, pp. 2355-2369. doi:10.1109/78.317857

[6] W. A. Gardner, A. Napolitano and L. Paura, "Cyclostationarity: Half a Century of Research," Signal Processing, Vol. 86, No. 4, April 2006, pp. 639-697. doi:10.1016/ j.sigpro.2005.06.016

[7] M. Simon and M.-S. Alouini, "Digital Communications over Fading Channels: A Unified Approach to Performance Analysis," John Wiley \& Sons, Hoboken, 2005.

[8] D. D. Klovsky, "Digital Data Transmission over Radio Channels," Sviaz, Moscow, 1982.

[9] A. Sendonaris, E. Erkip and B. Aazhang, "User Cooperation Diversity, Parts I and II," IEEE Transactions on Communications, Vol. 51, No. 11, November 2003, pp. 1928-1948. doi:10.1109/TCOMM.2003.819238

[10] V. Kontorovich and S. Primak, "Autocovariance Receiver for DTSM: Performance Characteristics for a Generalized MIMO Channel," Proceedings of the IWCMC'09, 19-22 June 2009, Leipzig.

[11] A. Papoulis, "Probability, Random Variables and Stochastic Processes," Mc-Graw Hill, New York, 1991.

[12] A. M. Hussein, "Multisensor Distributed Sequential Detection", IEEE Transactions on Aerospace and Electronic Systems, Vol. 30, No. 3, 1994, pp. 698-708. doi:10.1109/7. 303740

[13] R. Vishwantan and V. Aalo, "On Counting Rules in Distributed Detection," IEEE Transactions on Acoustic, Speech and Signal Proceedings, Vol. 37, No. 5, May 1989, pp. 772-775. doi:10.1109/29.17574

[14] V. V. Veeravalli, T. Basar and H. V. Poor, "Decentralized Sequential Detection with a Fusion Center Performing Sequential Tests," IEEE Transactions on Information Theory, Vol. 39, No. 2, March 1993, pp. 433-442, doi:10. $1109 / 18.212274$

[15] O. Filio, S. Primak and V. Kontorovich, "Impact of Scatering Environment on the Performance of Spectrum Sensing in Multiantenna Cognitive Radio Systems," Submitted to ICC 2011 Cognitive Radio and Network Symposium.

[16] D. Middleton, "A Statistical Theory of Reverberation and Similar First Order Scattered Fields," IEEE Transaction on Information Theory, Vol. 13, No. 3, July 1967, pp. 393414. doi:10.1109/TIT.1967.1054045

[17] P. Beckman and A. Spizzidino, "The Scattering of Electromagnetic Waves from Rough Surfaces," Pergamon Press, Oxford, 1963.

[18] I. S. Gradshteyn and I. M. Ryzhik, "Table of Integrals, Series and Products," Academic Press, Massachusetts, 2000 .

[19] M. Nakagami, "The M-Distribution: A General Formula of Intensity Distribution of Rapid Fadings," In: W. G. Hoffman, Ed., Statistical Methods in Radio Wave Propagation, Pergamon Press, Oxford, 1960.
[20] R. Zhang, T-J Lim et al., "Multiantenna Based Spectrum Sensing for Cognitive Radios: A GLRT Approach," IEEE Transactions on Communications, Vol. 58, No. 1, January 2010, pp. 84-88. doi:10.1109/TCOMM.2010.01.080158

[21] M. Shi, Y. Bar-Ness and W. Du, "A Simple Method to Enhance the Detection of Second Order Cyclostationarity," Proceedings of the GLOBECOM'08, New Orleans, 2008, pp. 1-6. doi:10.1109/GLOCOM.2008.ECP.679

[22] Y. Okunev, "Phase and Phase-Difference Modulation in Digital Communications," Artech-House, London, 1997.

[23] D. Middleton, "Introduction to the Statistical Theory of Communication," IEEE Press, 1999.

[24] K. Paulraj et al. "Introduction to Space-Time Wireless Communications," Cambridge University Press, Cambridge, 2006.

[25] V. Kontorovich and S. Primak, "Performance Analysis of OSTBC over Generalized Gaussian MIMO Channels," Proceedings of the IWCMC'09, Leipzig, 19-22 June 2009.

[26] A. Wald, "Sequential Analysis," Dover Publications, New York, 1973.

[27] Y. Zeng, Y.-C. Liang, et al., "A Review on Spectrum Sensing for Cognitive Radio: Challenges and Solutions," EURASIP Journal on Advances in Signal Processing Special Issue on Advanced Signal Processing for Cognitive Radio Networks, Vol. 2010, January 2010.

[28] N. H. Kamil and X. Yuan, "Detection Proposal Schemes for Spectrum Sensing in Cognitive Radio," Wireless Sensor Networks, Vol. 2, No. 5, 2010, pp. 365-372.

[29] Y. A. Goldstein and V. Ya. Kontorovich, "Noise Immunity of Weighted Discrete Addition of Diversity Signals," Telecommunications and Radio Engineering, Vol. 26/27, No. 11, 1972, pp. 104-106.

[30] R. Alvarez, H. Jardon and V. Kontorovich, "Micro-Diversity Properties of Cellular Radio Networks," Proceedings of Commsphere'95, Israel, 22-27January 1995, pp. 180-184.

[31] D. da Costa, et al., "An Improved Closed-Form Approximation to the Sum of Arbitrary Nakagami M Variates," IEEE Transactions on Vehicular Technology, Vol. 57, No. 6, 2008, pp. 3854-3863.

[32] Z. Khan, et al., "On the Selection of the Best Detection Performance Sensors for Cognitive Radio Networks," IEEE Signal Processing Letters, Vol. 17, No. 4, April 2010, pp. 359-362. doi:10.1109/LSP.2010.2041581

[33] V. Kontorovich, S. Primak, A. Alcocer-Ochoa and R. Parra-Michel, "MIMO Channel Orthogonalization Applying Universal Eigenbasis," IET Signal Processing, Vol. 2, No. 2, 2008, pp. 87-96. doi:10.1049/iet-spr:20070 126

[34] L. Ruan and V. K. N. Lau, "Power Control and Performance Analysis of Cognitive Radio Systems under Dynamic Spectrum Activity and Imperfect Knowledge of System State," IEEE Transactions on Wireless Communications, Vol. 8, No. 9, September 2009, pp. 4616-4622. doi:10.1109/ TWC.2009.080789 